\title{
HIV-related data among key populations to inform evidence-based responses: protocol of a systematic review
}

\author{
Amrita Rao ${ }^{1 *}$ DD, Sheree Schwartz ${ }^{1}$, Keith Sabin², Tisha Wheeler $^{3}$, Jinkou Zhao ${ }^{4}$, James Hargreaves ${ }^{5}$, \\ Stefan Baral ${ }^{1}$ and on behalf of the Global.HIV Research Group
}

\begin{abstract}
Background: Key populations who bear a disproportionate burden of HIV, including female sex workers, men who have sex with men, people who use drugs, transgender people, and incarcerated populations, have been understudied, especially in the context of broadly generalized HIV epidemics. Program and investment planning documents often do not take into account the data that do exist. Prior systematic reviews have been comprehensive, but lack sustainability and relevance over time. This review aims to synthesize all available data for key populations and present the data through an accessible, updatable user-friendly graphic interface. The outputs of this systematic review will serve as a resource for decision-makers, providing government stakeholders and donors with the tools to make evidence-based decisions for national planning.
\end{abstract}

Methods: We will conduct a systematic review of data published or made available between January 1, 2006, and January 1, 2019, that captures the burden of HIV, both prevalence and incidence estimates, HIV prevention and treatment cascades, key population size estimates, experienced violence, consistent condom use, and engagement with healthcare systems for female sex workers, men who have sex with men, people who use drugs, transgender people, and incarcerated populations. A team of reviewers will use Covidence to conduct two independent reviews of both title/abstract and full text for each article. REDCap will be used for data abstraction and storage.

Discussion: Findings from this systematic review and the development of the enhanced graphical interface to display data, along with ongoing efforts to build capacity among key stakeholders to better use and interpret available data, will help ensure that available epidemiologic data related to key populations can be appropriately used to guide largescale HIV funding and programmatic responses.

Systematic review registration: PROPSERO CRD42016047259.

Keywords: Key populations, Female sex workers, Men who have sex with men, People who use drugs, Incarcerated populations, Treatment cascade, Population size estimation, Systematic review

\section{Background}

As part of efforts to curtail the HIV pandemic and achieve an AIDS-free generation, international directives, including The Global Fund Strategy 2017-2022 and PEPFAR 3.0, have highlighted the need for quality data to inform an effective HIV response $[1,2]$. Understanding the scope, distribution, and determinants of

\footnotetext{
* Correspondence: arao24@jhu.edu

1 Department of Epidemiology, Johns Hopkins Bloomberg School of Public Health, 615 N. Wolfe St, Baltimore, MD 21205, USA

Full list of author information is available at the end of the article
}

country-level HIV epidemics, along with characterizing the effectiveness and ultimate impact of current programs, is needed to improve existing prevention and treatment service delivery systems [1,2]. For key populations who bear a disproportionate burden of HIV, including female sex workers, men who have sex with men, people who use drugs, transgender people, and incarcerated populations, defining this need has been particularly difficult [3]. Due to stigma, discrimination, and often criminalization, key populations are marginalized and hidden, resulting in greater risks of HIV acquisition

(c) The Author(s). 2018 Open Access This article is distributed under the terms of the Creative Commons Attribution 4.0 International License (http://creativecommons.org/licenses/by/4.0/), which permits unrestricted use, distribution, and 
and transmission [4-7]. However, these same determinants challenge characterizing the HIV epidemic within these populations and the potential impact on HIV epidemics among all reproductive aged adults. The needs of key populations are often understudied, particularly in high HIV burden settings, where less attention is often given to key populations based on assumptions of limited public health relevance [8-10]. In these generalized epidemic settings, there are often limited data available for female sex workers, gay men and other men who have sex with men, and people who use drugs, and even less data available for other key population groups including transgender women. Traditional approaches, including the static Modes of Transmission (MOT) model, have been used to predict the annual portion of new HIV infections acquired across subgroups. These traditional approaches to estimating the importance of the unmet treatment and prevention needs of key populations do not capture the longer chains of secondary indirect transmissions due to high-risk behaviors and are based solely on static HIV prevalence and assumed population size [11]. These approaches rarely account for underreporting of HIV-associated risks and also tend to treat key populations as isolated groups thereby ignoring the full chains of potential onward HIV transmission [11]. Recent dynamic transmission modeling suggests the importance of addressing the HIV prevention and treatment needs among key populations in all HIV epidemic settings [9, 11-13].

Where data are available for key populations, they are often underutilized in both official policy and program documents used by donors and policymakers $[9,14$, 15], possibly due to the lack of political will, the lack of access to relevant data, or the lack of knowledge around the specific issues facing key populations. In an examination of the utilization of key population size estimates in Global Fund and PEPFAR funding proposals and Country Operational Plans (COPs) [14], of the 71 population size estimate studies published between 2009 and 2016 only two were mentioned in Global Fund-related concept notes, 12 in PEPFAR COPs, and seven in national Ministry of Health documents [14]. Despite significant investments to conduct epidemiologic studies of key populations by these donors and an emphasis on the need for high-quality data to inform a response, there exists inadequate evidence of uptake of these data to guide the HIV response.

Overall, there are gaps both in the availability of quality data and utilization of the data of key populations disproportionately affected by HIV. In response to these gaps, the proposed study aims to complete and make available online, a systematic, living, and comprehensive review of all available data for key populations that can be used to inform an evidence-based and human rights affirming HIV response. Data will be reviewed, synthesized, and presented in the form of an enhanced graphical interface. The site will present data in a clear, user-friendly manner to facilitate its use by relevant stakeholders, such as donor governments and international bodies, local government officials, members of key population community leadership as well as affected members of the community, HIV researchers, academics, implementing partners, and the media.

\section{Methods/design}

We will conduct a systematic review of data published or made available between January 1, 2006, and January 1,2019 , that captures the burden and risk of HIV, both prevalence and incidence estimates, prevention indicators and treatment cascades, population size estimates, experienced violence, and engagement with healthcare systems. This protocol is registered in the PROPSERO database (CRD42016047259; 28 September 2016) and is in accordance with the guidelines specified in the Preferred Reporting Items for Systematic Reviews and Meta-analyses statement for protocols [16].

\section{Objective}

- To complete a global systematic review of all available data characterizing the burden of HIV and the HIV treatment cascade among key populations (female sex workers, men who have sex with men, people who use drugs, transgender people, and incarcerated populations) from 2006 to 2019.

- To conduct quality assessments for a subset of all data sources on key populations from 30 priority countries selected based on current HIV programming priorities, on prevalence, incidence, treatment cascades, and population size estimates using a quality assessment tool developed for assessing key populations data.

\section{Primary outcomes}

1. Burden of HIV among key populations as characterized by prevalence and incidence of HIV from 2006 to 2019.

2. HIV treatment cascade among key populations from 2006 to 2019.

Secondary outcomes include prevention indicators (Engaged in HIV testing, knowledge of HIV prevention, condom and PrEP availability, consistent condom use), population size estimates (including specific subnational organizational units to which they apply), experienced violence (physical, sexual, intimate partner), and engagement with healthcare systems for key 
populations [female sex workers, men who have sex with men, people who use drugs, transgender people, and incarcerated populations], and to make these data updatable and available in the form of an enhanced graphical interface.

\section{Information sources and search strategies}

In partnership with information management specialists at Johns Hopkins University, we will search the following

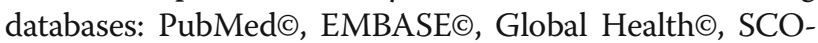

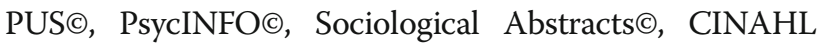
(Cumulative Index to Nursing and Allied Health Literature) $\odot$, Web of Science@, and POPLine $\odot$.

Peer-reviewed conference abstracts will be searched from online publications of conference proceedings, including those of International AIDS Conference (IAC), the Conference on HIV Pathogenesis, Treatment, and Prevention, HIV Research for Prevention (HIVR4P), and the Conference on Retroviruses and Opportunistic Infections (CROI). The World Health Organization (WHO) publications database will be also searched as well as the National Library of Medicine's Meeting Abstracts database (https://wwwcf.nlm.nih.gov/hsr_project/home_proj.cfm).

Other data sources from the gray literature will be identified through the Development Experience Clearinghouse, including national surveillance system data reports for example Demographic Health Surveys and Integrated Biological and Behavioral Surveys, as well as studies conducted by large international non-governmental organizations. The Clearinghouse will be systematically searched. These reports have been previously synthesized from global reporting databases, Global Fund grant application documents, and the gray literature [17].

Search strategies were developed based on a combination of controlled vocabulary (e.g., $\mathrm{MeSH}$ terms) and other keyword searches. These search strategies were adapted from existing search strategies developed for earlier systematic reviews of key populations [5, 18-20]. Multiple iterations of the search strategies were piloted in order to prioritize a highly sensitive search that captured all available data for key populations and HIV. The search strategies are made up of search terms for three independent concepts. Concept one is made up of terms for the population of interest, concept two is made up of terms for HIV, and concept three is made up of terms for violence. Each search run is a combination of concept one AND (concept two OR concept three). Detailed search strategies are available in Additional file 1.

\section{Inclusion and exclusion criteria}

To be included in the review, data must meet the following criteria:
1. Studies of any design that include either HIV data or violence data among female sex workers, men who have sex with men, people who use drugs, transgender populations, and incarcerated populations, even if these groups are not the main focus of the study.

2. Participants in studies can be of any age, race, or ethnicity.

3. Data must be published in a peer-reviewed journal, presented as an abstract at a scientific conference, or available on the web from governmental or nongovernmental sources.

4. Qualitative studies and modeling studies will be included as searchable records in the data repository, but qualitative data will be not abstracted or included in the quantitative narrative analysis.

5. Published or presented between January 1, 2006, and January 1, 2019.

6. Data from all countries and settings will be included.

The following types of studies will be excluded from the review:

1. Studies where the sample size was less than 50 .

2. Studies published in languages other than English, French, and Spanish.

\section{Screening and selection}

Titles, abstracts, citation information, and descriptor terms of citations identified through the search strategy will be screened by a team of reviewers and will be selected to move to full-text review if there is reason to believe that the above six criteria are met. Full-text articles will be obtained of all selected abstracts and the team of reviewers will conduct two independent reviews of each article to assess all full-text articles for eligibility to determine final study selection. The same set of questions will be used for full-text screening. Title/abstract and full-text review will be conducted in Covidence, a tool designed to help facilitate the systematic review process, produced in partnership with Cochrane Reviews. Differences will be resolved by a third independent reviewer.

\section{Data abstraction and data collection process}

Data will be abstracted independently by a team of reviewers using standardized data abstraction forms in REDCap. Differences in data abstraction will be resolved using REDCap's data comparison tool by a third, independent reviewer. Reviewers will be trained using the data collection tool on how to abstract available information from eligible articles and how to index the article in the database. While not all articles will have 
information for all indicators being captured in the REDCap tool, reviewers will complete the data tool based on available data. The REDCap tool outlining data capture of outcomes is available in Additional file 2.

The following information will be gathered from each included study:

- Study identification: author(s); citation; year of publication

- Study description: location, setting, population; years (period of study); study design; sample size; age range; sex and gender, if reported separately

- Outcomes (specified above)

The review and corresponding data repository will be updated regularly. The plan for updating is to run automated searches based on the same search strategies originally developed once every 6 months, following the end date of the initial review. The first update will be done in June 2019 to include articles published between January and June 2019. Data review and entry will be done on a rolling basis.

Amendments to the protocol originally published in PROSPERO include the following:

- The available data and outcomes have been more precisely specified.

- Articles published in other languages including French and Spanish will be reviewed.

- The quality assessment tool developed is a novel tool rather than a modified Downs and Black checklist.

\section{Quality assessments}

A quality assessment tool has been developed and adapted for key populations research from the NHLBI Quality Assessment Tool for Observational Cohort and Cross-Sectional Studies [21, 22]. The tool was adapted by the study team in order to capture the essential elements of the original tool, while also ensuring applicability to our main outcomes. The tool is designed such that two independent assessors evaluate first the general study design and the study implementation and then evaluate outcome-specific data quality. The quality assessment tool is designed to assess the quality of available evidence for data points of HIV prevalence, incidence, the HIV care continuum, and population size estimates. Data points will be categorized as "good," "fair," or "poor" based on an evaluation of a number of criteria related to study design, study implementation, and use of appropriate analytic methods.

The quality assessment tool will be applied to available data points for 30 priority countries, selected from the larger review because they have significant bilateral and multilateral support for HIV programs. The choice of 30 priority countries is based on current donor priorities-where resources are invested, where current programs for key populations and HIV exist, and where more data are needed to inform programs. As a starting point, therefore, we wanted to focus our efforts on conducting quality assessments in countries where the assessments would be used immediately.

All articles and reports available for a specific country will be gathered and efforts will be taken to group publications based on the study of origin. Papers from the same original study will be reviewed together to evaluate the quality of the reported data emanating from the single study.

The quality assessment tool is available in Additional file 3.

\section{Evidence synthesis}

The primary analysis will involve looking at a synthesis of the burden of HIV and the HIV treatment cascade among key populations living with HIV. We will investigate comparisons by population and by country, in particular focusing on comparisons within sub-Saharan Africa and regions within sub-Saharan Africa. We anticipate heterogeneity of the data and therefore propose a narrative synthesis. If feasible, we will consider including a meta-analysis. Secondary analyses will involve looking at reporting of pre-exposure prophylaxis (PrEP) use and PrEP uptake among those who are HIV negative. Similarly, this will involve comparisons across populations and countries and will involve a narrative synthesis. Additional secondary analyses involving prevention indicators (engaged in HIV testing, knowledge of HIV prevention, condom availability, consistent condom use), population size estimates, experienced violence (physical, sexual, intimate partner), and engagement with healthcare systems for key populations may be proposed at a later date.

\section{Dissemination}

In response to limitations and challenges in using data from previous reviews, we determined there will be added value to capture all of the data reviewed and included for this review in a database that is accessible and updatable. The results will therefore be used to generate a data repository for key populations that will be integrated into an online, graphical interface that will display global HIV data synthesized during the systematic review. The online global map will visually display all available data, will allow for comparison of key HIV statistics among different countries, will provide country dashboard pages that highlight key information, and will allow for datasets to be downloaded by researchers, government officials, community-based organizations, members of the community, and the general public. 


\section{Discussion}

Despite evidence of the importance and overall impact of prioritizing key populations in implementing effective and efficient HIV responses, many countries have limited current data or no data characterizing the unmet needs of key populations [9-13, 23, 24]. Where data are available, they are often not used in key documents and policy decisions, despite an international call to further evidence-based policies [14]. Systematic reviews conducted in the past among key populations have comprehensively identified where data exist and synthesized available data to provide estimates on a range of indicators, including HIV prevalence, HIV incidence, and population size estimates $[5,6,14,18,19,25-30]$. However, with rapidly evolving methods for estimating population size and HIV transmission dynamics, these estimates have become dated both in terms of age of data and their usability by key stakeholders. Moreover, given persistent stigma and ever-changing laws and policies related to the criminalization of key populations including laws focused on same-sex practices, sex work, gender identity, and injection drug use, the need for current and local level estimates is crucial for key populations [31]. To this end, the current review, online platform, and plans for ongoing and regular updates will make responding to changing contexts and environments more feasible in real-time.

Past reviews have synthesized data to produce pooled estimates at the national or global level. This level of analysis is helpful for understanding the unmet needs for key populations, but accessing and critically assessing individual data points can help better inform the decision of the utility of these data for informing policy. The current review is intended to be flexible, sustainable, and relevant over time, with updates to the global interactive tool scheduled to happen on a regular basis. Key stakeholders will have the ability to make data requests that specify the populations, countries, or indicators in which they have the interest or need. These data pulls will contain key study design information where available and quality assessments so that those using the data have the relevant information they need to make informed decisions about data use.

We have identified several challenges in designing and conducting this updatable systematic review of global key population data. First, capturing information from a large pool of sources from disparate fields of study, among different populations, and in different countries required us to make decisions on what information we could feasibly capture. As an example, consistent condom use is often an important indicator or predictor for assessing level of sexual transmission of HIV, risk for other STIs, partner dynamics, and uptake of interventions designed to improve condom use.
Consistent condom use, however, is not systematically defined. How a study or report defines consistent condom use can vary in terms of time period [e.g., condoms used during the last act, the last week, the last 30 days], frequency [e.g., always/sometimes/never, number of times], and partner type [e.g., with all partners, disaggregated by clients/regular/casual], etc. In addition, there are differences in how much is specified related to what "condom use" even means, that is was one "ever" used, was it used for the "whole duration of the act", was it "reused." We wanted to capture consistent condom use but had to account for differences in reporting. To be able to capture consistent condom use then, we decided to capture the proportion who reported consistent condom use however the study or report defined it, but to have an additional indicator allowing the data entrant to specify what exactly "consistent condom use" meant for each data point. Another challenge we identified was dealing with multiple studies or reports published from the same study. Because our unit of analysis in this systematic review was the data source, it was important to determine a strategy to account for duplication of certain data points. To do this, we created a cohort identifier based on key information that would apply to the study itself rather than the publication or report in order to have the ability to later identify which sources were actually referring to the same data point. Including all of the estimates would bias our results towards those studies with the most publications. Finally, in order to create a user-friendly online resource for collating and displaying global HIV data for key populations, we needed to make decisions on how to visualize data on a range of indicators, including prevalence, incidence, the treatment cascade, and population size estimates. There are multiple levels to the data that are being synthesized: key population group, country, and indicator of interest, and creating a system that would not only display the available data in a clear, understandable way, but also be flexible and updatable, continues to be a challenge. With the support of our web development team and the infrastructure of Carto, we have been able to accommodate these different levels of data and allow for ease of use. We chose to use an interactive global map that allows the user to select key indicators to geographically display, provides country-specific dashboards with key information and indicators highlighted for different key population groups, and creates data visualization graphs showing changes in key indicators over time and comparisons across countries. The value in conducting this large systematic review, with a range of indicators, is that it will allow us to go beyond standard single population systematic reviews of key population groups, giving us the ability to assess overlap of populations (e.g., female sex worker who injects drugs) and make comparisons between groups on key indicators. This will also allow for comprehensive 
identification of data gaps or unmet needs among key populations and prioritization of funding for individual populations based on the context-specific needs within a single country or region. Findings from this systematic review and the development of the enhanced graphical interface to display data will help ensure that available epidemiologic data related to key populations can be more effectively used in real-time to inform HIV-related policies and programs.

\section{Additional files}

\section{Additional file 1: Provides detailed search strategies by database.} (DOCX $27 \mathrm{~kb}$ )

Additional file 2: Provides the data collection tool for abstraction of a range of study specific details and HIV and violence related indicators for key populations. (PDF $321 \mathrm{~kb}$ )

Additional file 3: Provides the quality assessment tool used to evaluate data points for prevalence and incidence of HIV, the treatment cascade, and population size estimates. (DOCX $32 \mathrm{~kb}$ )

\section{Abbreviations}

AIDS: Acquired immunodeficiency syndrome; COPs: Country Operational Plans; CROI: Conference on Retroviruses and Opportunistic Infections; HIV: Human immunodeficiency virus; HIVR4P: HIV Research for Prevention; IAC: International AIDS Conference; MeSH: Medical subject headings; MOT: Modes of transmission; NHLBI: National Heart, Lung, and Blood Institute; PEPFAR: President's Emergency Plan for AIDS Relief; PrEP: Preexposure prophylaxis; STIs: Sexually transmitted infections; WHO: World Health Organization

\section{Acknowledgements}

Thank you very much to Lori Rosman, Informationist at Johns Hopkins, without whom this review would not be possible.

The Global.HIV research group is made up of the following members:

Zafir Abutalib

Chase Alston

Joe Amoah

Anna Bickers

Ashley Charest

Meghan Holtzman

Cameron Meade

Lookman Mojeed

Madeline Nelson

Albert Osei

Mariela Pinedo

Summer Rak

Arlene Reich

Yvonne Robles

Kavya Sanghavi

Alex Schmall

Owen Stokes-Cawley

Vinithra Varadarajan

Kia Vaughn

Nikita Viswasam

Dexter Waters

\section{Funding}

The Study was made possible through the Project SOAR Cooperative Agreement AID-OAA-13-00060. Project SOAR is made possible by the generous support of the American people through the President's Emergency Plan for AIDS Relief and the United States Agency for International Development (USAID).

\section{Availability of data and materials}

The data repository generated during this study will be available upon completion of the review and generation of the graphical interface at global.hiv.

\section{Authors' contributions}

AR was responsible for developing the search protocol and strategies, helped with the conception of the data repository and online tool, and took the lead role in the writing of the manuscript. SS provided valuable feedback on the content and structure of the manuscript and played a major role in the conception of the study, the data repository, the online tool, and the quality assessments. KS provided valuable feedback on the content and structure of the manuscript and in the conception of the study, repository, and quality assessments. TW provided valuable feedback on the content and structure of the manuscript and guidance in the development of the study, the data repository, the online tool, and the quality assessments. JZ provided valuable feedback on the content and structure of the manuscript. JH provided valuable feedback on the content and structure of the manuscript. SB provided valuable feedback on the content and structure of the manuscript and played a major role in the conception of the study, the data repository, the online tool, and the quality assessments. All authors read and approved the final manuscript.

Ethics approval and consent to participate

Not applicable.

\section{Consent for publication}

Not applicable.

\section{Competing interests}

The authors declare that they have no competing interests.

\section{Publisher's Note}

Springer Nature remains neutral with regard to jurisdictional claims in published maps and institutional affiliations.

\section{Author details \\ ${ }^{1}$ Department of Epidemiology, Johns Hopkins Bloomberg School of Public Health, 615 N. Wolfe St, Baltimore, MD 21205, USA. Strategic Information and Evaluation, Joint United Nations Programme on HIV/AIDS, Geneva, Switzerland. ${ }^{3}$ Office of HIV/AIDS, United States Agency for International Development, Washington, DC, USA. ${ }^{4}$ Technical Advice and Partnerships Department, The Global Fund to fight AIDS, Tuberculosis, and Malaria, Geneva, Switzerland. ${ }^{5}$ Department of Social and Environmental Health, London School of Hygiene and Tropical Medicine, London, UK.}

Received: 13 July 2018 Accepted: 22 November 2018

Published online: 03 December 2018

\section{References}

1. PEPFAR 3.0. Office of the US Global AIDS Coordinator. Available from: http:// www.pepfar.gov/documents/organization/234744.pdf.

2. The Global Fund Strategy 2017-2022: Investing to end epidemics. The Global Fund to Fight AIDS, Tuberculosis and Malaria. Available from: https://www.theglobalfund.org/media/2531/core_globalfundstrategy20172022_strategy_en.pdf..

3. UNAIDS. The gap report. Geneva, Switzerland; 2014.

4. Kim HY, Grosso A, Ky-Zerbo O, Lougue M, Stahlman S, Samadoulougou C, et al. Stigma as a barrier to health care utilization among female sex workers and men who have sex with men in Burkina Faso. Ann Epidemiol. 2018;28(1):13-9.

5. Baral S, Beyrer C, Muessig K, Poteat T, Wirtz AL, Decker MR, et al. Burden of HIV among female sex workers in low-income and middle-income countries: a systematic review and meta-analysis. Lancet Infect Dis. 2012; 12(7):538-49.

6. Fitzgerald-Husek A, Van Wert MJ, Ewing WF, Grosso AL, Holland CE, Katterl $\mathrm{R}$, et al. Measuring stigma affecting sex workers (SW) and men who have sex with men (MSM): a systematic review. PLoS One. 2017;12(11):e0188393.

7. Stangl AL, Lloyd JK, Brady LM, Holland CE, Baral S. A systematic review of interventions to reduce HIV-related stigma and discrimination from 2002 to 2013: how far have we come? J Int AIDS Soc. 2013;16(3 Suppl 2):18734.

8. Pettifor A, Rosenberg N, Behets F. The need to focus on sex workers in generalized HIV epidemic settings. Sex Transm Dis. 2011;38(4):324-5.

9. Mishra S, Boily MC, Schwartz S, Beyrer C, Blanchard JF, Moses S, et al. Data and methods to characterize the role of sex work and to inform sex work 
programs in generalized HIV epidemics: evidence to challenge assumptions. Ann Epidemiol. 2016;26(8):557-69.

10. Boily MC, Pickles M, Alary M, Baral S, Blanchard J, Moses S, et al. What really is a concentrated HIV epidemic and what does it mean for West and Central Africa? Insights from mathematical modeling. J Acquir Immune Defic Syndr. 2015:68(Suppl 2):S74-82.

11. Mishra S, Pickles M, Blanchard JF, Moses S, Shubber Z, Boily MC. Validation of the modes of transmission model as a tool to prioritize HIV prevention targets: a comparative modelling analysis. PLoS One. 2014;9(7):e101690.

12. Mishra S, Pickles M, Blanchard JF, Moses S, Boily MC. Distinguishing sources of HIV transmission from the distribution of newly acquired HIV infections: why is it important for HIV prevention planning? Sex Transm Infect. 2014;90(1):19-25.

13. Mishra S, Steen R, Gerbase A, Lo YR, Boily MC. Impact of high-risk sex and focused interventions in heterosexual HIV epidemics: a systematic review of mathematical models. PLoS One. 2012;7(11):e50691.

14. Baral SD, Lyons C, Sullivan E, Kurani S, Sherwood J, Millett G, et al. The uptake of population size estimation studies for key populations in guiding HIV responses across sub-Saharan Africa: a systematic review. Durban, South Africa: AIDS 2016; 2016

15. Belay T, Mbuya N, Rajan V. Data utilization and evidence-based decision making the health sector: survey of three Indian States. South Asia human development sector series: World Bank; 2009. p. 27.

16. Moher D, Liberati A, Tetzlaff J, Altman DG, Group TP. Preferred Reporting Items for Systematic Reviews and Meta-Analyses: The PRISMA Statement. PLoS Med. 2009;6(7):e1000097.

17. Sabin K, Zhao J, Garcia Calleja JM, Sheng Y, Arias Garcia S, Reinisch A, et al. Availability and quality of size estimations of female sex workers, men who have sex with men, people who inject drugs and transgender women in low- and middle-income countries. PLoS One. 2016;11(5):e0155150.

18. DeBeck K, Cheng T, Montaner JS, Beyrer C, Elliott R, Sherman S, et al. HIV and the criminalisation of drug use among people who inject drugs: a systematic review. Lancet HIV. 2017;4(8):e357-e74.

19. Baral SD, Poteat T, Stromdahl S, Wirtz AL, Guadamuz TE, Beyrer C Worldwide burden of HIV in transgender women: a systematic review and meta-analysis. Lancet Infect Dis. 2013;13(3):214-22.

20. Beyrer C, Baral SD, van Griensven F, Goodreau SM, Chariyalertsak S, Wirtz AL, et al. Global epidemiology of HIV infection in men who have sex with men. Lancet. 2012;380(9839):367-77.

21. Quality Assessment Tool for Observational Cohort and Cross-Sectional Studies [Internet]. National Heart. In: Lung and blood institute; 2017.

22. Downs SH, Black N. The feasibility of creating a checklist for the assessment of the methodological quality both of randomised and non-randomised studies of health care interventions. J Epidemiol Community Health. 1998;52:377-84.

23. Boily MC, Pickles M, Lowndes CM, Ramesh BM, Washington R, Moses $\mathrm{S}$, et al. Positive impact of a large-scale HIV prevention programme among female sex workers and clients in South India. AIDS. 2013;27(9):1449-60.

24. Freeman EE, Orroth KK, White RG, Glynn JR, Bakker R, Boily MC, et al. Proportion of new HIV infections attributable to herpes simplex 2 increases over time: simulations of the changing role of sexually transmitted infections in sub-Saharan African HIV epidemics. Sex Transm Infect. 2007; 83(Suppl 1):i17-24.

25. Baral S, Sifakis F, Cleghorn F, Beyrer C. Elevated risk for HIV infection among men who have sex with men in low- and middle-income countries 20002006: a systematic review. PLoS Med. 2007;4(12):e339.

26. MacCarthy S, Poteat T, Xia Z, Roque NL, Hyun Jin Kim A, Baral S, et al. Current research gaps: a global systematic review of HIV and sexually transmissible infections among transgender populations. Sex Health. 2017;14(5):456-68.

27. Papworth E, Ceesay N, An L, Thiam-Niangoin M, Ky-Zerbo O, Holland C, et al. Epidemiology of HIV among female sex workers, their clients, men who have sex with men and people who inject drugs in West and Central Africa. J Int AIDS Soc. 2013;16(Suppl 3):18751.

28. Poteat T, Scheim A, Xavier J, Reisner S, Baral S. Global epidemiology of HIV infection and related syndemics affecting transgender people. J Acquir Immune Defic Syndr. 2016;72(Suppl 3):S210-9.

29. Stromdahl S, Hickson F, Pharris A, Sabido M, Baral S, Thorson A. A systematic review of evidence to inform HIV prevention interventions among men who have sex with men in Europe. Euro Surveill. 2015; 20(15):21096.
30. Sullivan PS, Jones JS, Baral SD. The global north: HIV epidemiology in highincome countries. Curr Opin HIV AIDS. 2014;9(2):199-205.

31. Schwartz SR, Nowak RG, Orazulike I, Keshinro B, Ake J, Kennedy S, et al. The immediate eff ect of the same-sex marriage prohibition act on stigma, discrimination, and engagement on HIV prevention and treatment services in men who have sex with men in Nigeria: analysis of prospective data from the TRUST cohort. Lancet HIV. 2015;2(7):e299-306.
Ready to submit your research? Choose BMC and benefit from:

- fast, convenient online submission

- thorough peer review by experienced researchers in your field

- rapid publication on acceptance

- support for research data, including large and complex data types

- gold Open Access which fosters wider collaboration and increased citations

- maximum visibility for your research: over $100 \mathrm{M}$ website views per year

At BMC, research is always in progress.

Learn more biomedcentral.com/submissions 